\title{
Comparison of two preoperative positioning techniques and unpositioning methods in non-intubated video-assisted thoracoscopic surgery
}

\author{
Yang Yu ${ }^{1 \#}$, Rong-Sheng Liu ${ }^{2 \#}$, Yun Bo ${ }^{3 \#}, \mathrm{Jia} \mathrm{Ye}^{2}$, Yu Huang ${ }^{2}$, Zhen-Zhen Xu' ${ }^{1}$ Zhi-Yan Yang ${ }^{2}$, \\ Zhi-Ming Chen ${ }^{4}$, Jun Peng ${ }^{1}$
}

${ }^{1}$ Department of Thoracic Surgery, The First People's Hospital of Yunnan Province, The Affiliated Hospital of Kunming University of Science and Technology, Kunming, China; ${ }^{2}$ Medical School, Kunming University of Science and Technology, Kunming, China; ${ }^{3}$ Department of Anesthesiology, The First People's Hospital of Yunnan Province, The Affiliated Hospital of Kunming University of Science and Technology, Kunming, China; ${ }^{4}$ Department of Radiology, The First People's Hospital of Yunnan Province, The Affiliated Hospital of Kunming University of Science and Technology, Kunming, China

Contributions: (I) Conception and design: Y Yu, RS Liu, Y Bo, ZM Chen, J Peng; (II) Administrative support: Y Yu, RS Liu, Y Bo, ZM Chen, J Peng; (III) Provision of study materials or patients: Y Yu, RS Liu, Y Bo, ZZ Xu, ZM Chen, J Peng; (IV) Collection and assembly of data: Y Yu, RS Liu, Yu Huang, Y Ye, ZY Yang; (V) Data analysis and interpretation: Y Yu, RS Liu, Y Bo, ZM Chen, J Peng; (VI) Manuscript writing: All authors; (VII) Final approval of manuscript: All authors.

"These authors contributed equally to this work.

Correspondence to: Jun Peng. Department of Thoracic Surgery, The First People's Hospital of Yunnan Province, The Affiliated Hospital of Kunming University of Science and Technology, Kunming, China. Email: 389647518@qq.com; Zhi-Ming Chen. Department of Radiology, The First People's Hospital of Yunnan Province, The Affiliated Hospital of Kunming University of Science and Technology, Kunming, China. Email: Zmchenyn@163.com.

Background: Using the non-intubated video-assisted thoracoscopic surgery (VATS) approach for small pulmonary nodules (SPNs) can accelerate patients' postoperative recovery. However, locating the SPNs intraoperatively by palpation can be difficult for thoracic surgeons. The advantages of using different preoperative positioning materials are different, especially for pulmonary-nodule-location-needle (P-NL-N) and the microcoil. This retrospective study analyzed the advantages of two preoperative positioning techniques for VATS under non-intubation anesthesia.

Methods: The data were collected for a total of 150 patients with pulmonary nodules who underwent nonintubated VATS at the First People's Hospital of Yunnan Province from January 2018 to January 2021. The patients were divided into a preoperative positioning group (including a P-N-L-N group and microcoil group) and an unlocalized group. These included patients were all compliant with surgical guidelines and were suitable for preoperative localization. Their intraoperative and postoperative indicators were compared, and among these indicators, the operative time, number of postoperative drainage days, postoperative total drainage volume, postoperative discharge time was efficacy group and the intraoperative blood loss was safety group.

Results: Preoperative localization helped surgeons to explore nodules faster intraoperatively and remove SPNs precisely under non-intubated VATS. But the advantages of using different preoperative positioning materials are different. Positioning with either microcoil or P-N-L-N resulted in less operation time (P-NL-N group: $94.90 \pm 28.42 \mathrm{~min}$, microcoil group: $112.80 \pm 28.6 \mathrm{~min}, \mathrm{P}<0.05$ ), less intraoperative blood loss (P-N-L-N group: $35.80 \pm 21.17 \mathrm{~mL}$, microcoil group: $75.00 \pm 65.22 \mathrm{~mL}, \mathrm{P}<0.001$ ) and less postoperative thoracic drainage volume (P-N-L-N group: $64.90 \pm 181.96 \mathrm{~mL}$, microcoil group: $648.52 \pm 708.81 \mathrm{~mL}$, $\mathrm{P}<0.001)$. However, the postoperative discharge time $(\mathrm{P}-\mathrm{N}-\mathrm{L}-\mathrm{N}$ group: $5.02 \pm 1.35$ days, microcoil group: 5.40 \pm 2.79 days, $\mathrm{P}=0.38)$ and postoperative drainage time( $\mathrm{P}-\mathrm{N}-\mathrm{L}-\mathrm{N}$ group: $2.58 \pm 1.70$ days, microcoil group: $3.18 \pm 2.49$ days, $\mathrm{P}=0.16$ ) was not statistically significant. Positioning with $\mathrm{P}-\mathrm{N}-\mathrm{L}-\mathrm{N}$ seemed to have a better auxiliary effect for non-intubated VATS, suggesting its use can assist surgeons to determine the location of 
the lesion more accuracy intraoperatively. There was no significant difference in the pathological results among the groups.

Conclusions: Localization of SPNs is beneficial in non-intubated VATS, and the use of P-N-L-N was more effective than the microcoil in reducing operative time, intraoperative blood loss, postoperative total drainage volume, and postoperative discharge time.

Keywords: Microcoil; non-intubated video-assisted thoracoscopic surgery (non-intubated VATS); preoperative positioning; pulmonary-nodules-location-needle (P-N-L-N)

Submitted Jan 14, 2022. Accepted for publication Feb 14, 2022.

doi: $10.21037 /$ jtd-22-114

View this article at: https://dx.doi.org/10.21037/jtd-22-114

\section{Introduction}

With the popularization of low-dose computed tomography (CT) imaging in clinical practice, more and more small pulmonary nodules (SPNs) are being detected (1), which can lead to the discovery of lung cancer (2). At present, the preferred method for the early diagnosis and treatment of pulmonary nodules is video-assisted thoracoscopic surgery (VATS) (3). Thoracic pneumonectomy is performed under conventional tracheal intubation anesthesia, but tracheal intubation is an invasive procedure that can damage the trachea, cause postoperative discomfort in the throat of the patient, and also cause damage to the non-ventilated lung (4). Moreover, it requires a large dose of anesthetics. Nonintubated VATS reduces the above factors and it can reduce the pain caused by endotracheal intubation to patients (5). However, in non-intubated VATS, if the operation time is too long, the cognitive function and postoperative recovery of the patient is badly affected (6,7). In addition, intraoperative exploration and accurate surgical resection of the SPNs themselves can be difficult. Therefore, preoperative localization of the SPNs is very important for smooth nonintubated VATS and accurate tumor resection, especially for nodules with a small diameter, low density and located distant from the pleural edge.

There are various preoperative positioning techniques for SPNs, the commonly used clinical positioning techniques include hook wire positioning, methylene blue puncture positioning, bio-gel positioning and electromagnetic navigation bronchoscopy (8). However, the use of Hook Wire localization is likely to cause complications such as pulmonary hemorrhage, hemoptysis, chest wall hematoma, pneumothorax and even air embolism, in addition, positioning with methylene blue Punctur, Bio-gel and electromagnetic navigation bronchoscopy is time-consuming and can easily lead to blurred boundaries between SPNs and surrounding lung tissue (9). At present, the more commonly used positioning technology is pulmonary-nodule-locationneedle (P-N-L-N) or microcoil positioning, but the structure and shape of these two positioning materials are completely different, so they have different advantages. We present the following article in accordance with the STROBE reporting checklist (available at https://jtd. amegroups.com/article/view/10.21037/jtd-22-114/rc).

\section{Methods}

\section{Clinical data}

A total of 150 patients with pulmonary nodules who underwent non-intubated VATS in the Department of Thoracic Surgery of the First People's Hospital of Yunnan Province from January 2018 to January 2021 were enrolled. For SPNs patients, preoperative localization or nonlocalization as well as two different localization techniques during localization showed different results, so the unlocalized patients were included in this study. Therefore, they were divided into three groups: 50 patients who underwent CT-guided puncture of the SPNs and placement of a locating needle (Ningbo Sheng Jie Kang Biological Technology Co., Ltd., Ningbo, China) comprised the pulmonary-nodule-location-needle (P-N-L-N) group; 50 patients who underwent CT-guided puncture of the SPNs and placement of a 0.018 -in micro spring coil (specifications: $8 \mathrm{~mm} \times 2 \mathrm{~mm}, 6 \mathrm{~mm} \times 2 \mathrm{~mm}, 5 \mathrm{~mm} \times 2 \mathrm{~mm}$; Cook Company, USA) comprised the microcoil group; other 50 patients who did not localization of the pulmonary nodules before pneumonectomy were classified as the unlocalized group. The basic clinical characteristics of all patients are shown in Table 1. 
Table 1 Clinical characteristics of the patients

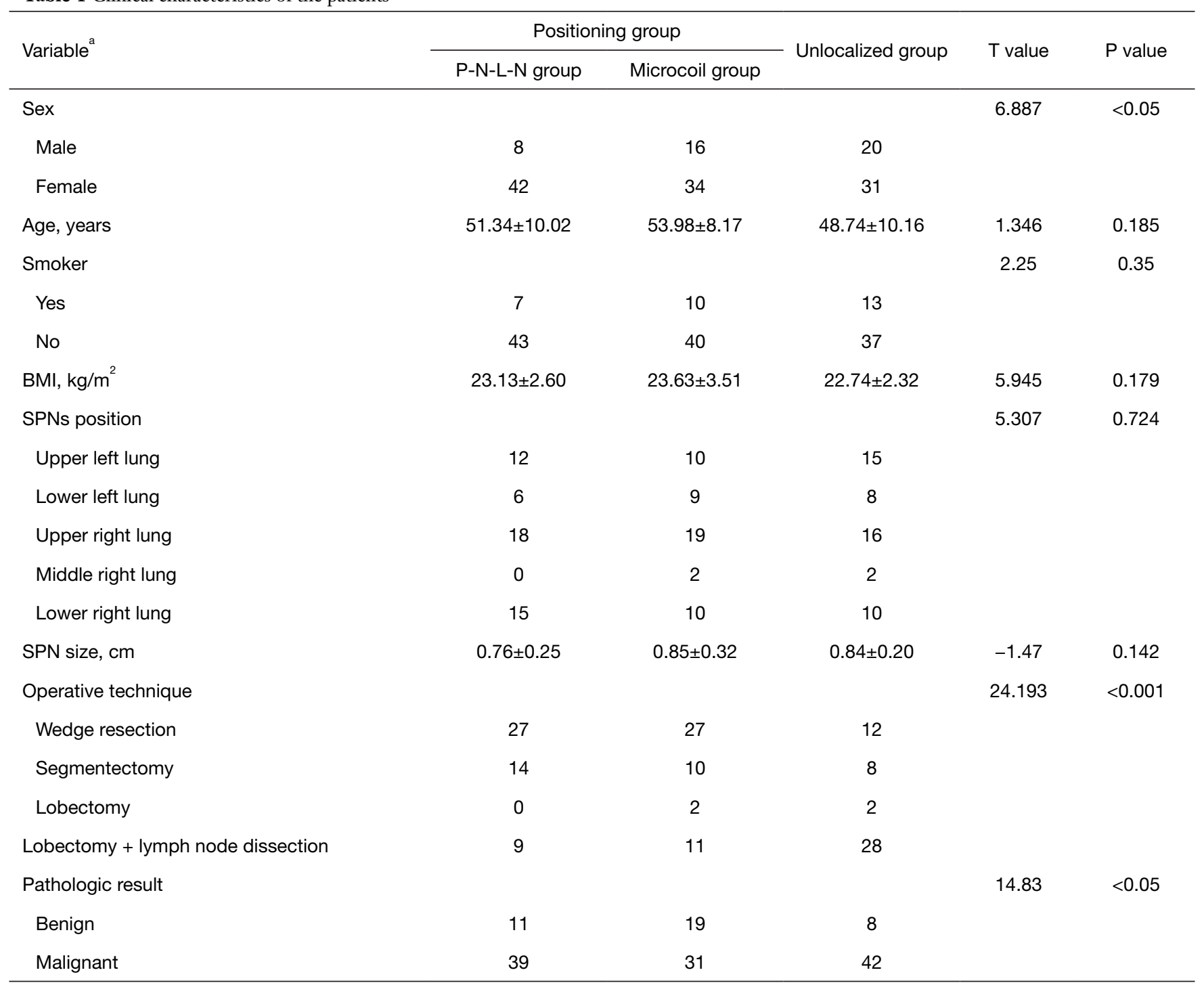

a , continuous data are shown as mean \pm standard deviation. BMI, body mass index; P-N-L-N, pulmonary-nodule-location-needle; SPNs, small pulmonary nodules.

\section{Inclusion criteria}

The inclusion criteria for the three groups were as follows: (I) Location group patients agreed to preoperative localization before undergoing VATS, (II) maximum diameter of SPNs $<2.0 \mathrm{~cm}$, and imaging features suspicious for malignant nodules, (III) patient was born in an area with a high incidence of lung cancer or had a family history of lung cancer, (IV) no contraindications to surgery, and (V) American Standards Association class I or II. Ethical approval for the study was granted by the Ethics Committee of The First People's Hospital of Yunnan Province (No.
KHLL2021-KY091). In addition, written informed consent was obtained from all patients who participated in the study. All procedures performed in this study involving human participants were in accordance with the Declaration of Helsinki (as revised in 2013).

\section{Exclusion criteria}

The exclusion criteria for the three groups was essentially the same: (I) patient chose regular review and declined operation, (II) maximum diameter of SPNs was $>2.0 \mathrm{~cm}$, and 


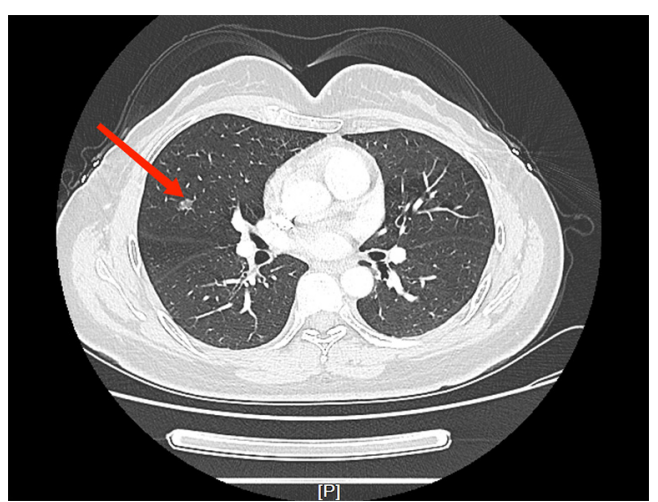

Figure 1 A small pulmonary nodule with small diameter, low density and located distant from the pleural edge. The red arrows indicate the SPNs that need to be located. SPNs, small pulmonary nodules.

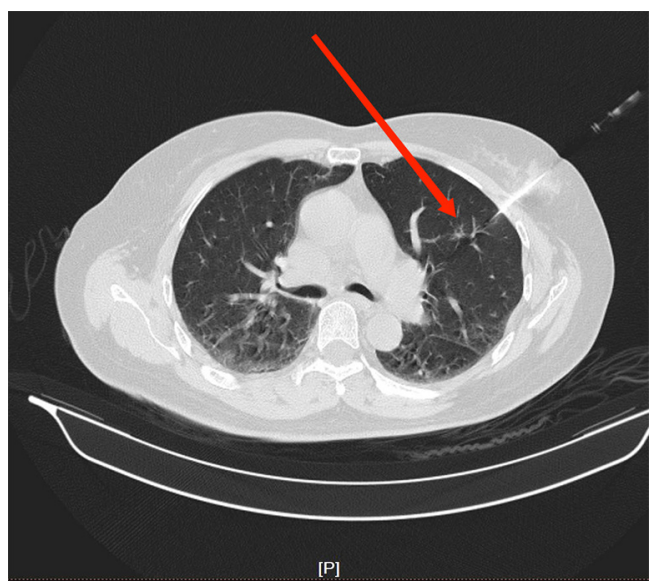

Figure 2 Localization of a nodule using the P-N-L-N. The red arrow shows the location of SPNs. P-N-L-N, pulmonary-nodulelocation-needle; SPNs, small pulmonary nodules.

the imaging features suggested benign nodules, (III) patients with thyroid dysfunction, blood system diseases, coronary heart disease and other serious primary diseases, and (IV) a history of difficulty with intubation or BMI $\geq 30 \mathrm{~kg} / \mathrm{m}^{2}$.

\section{Positioning}

According to the preoperative CT images, for SPNs with small diameter, low density and distant from the pleural edge (Figure 1), the interventional radiologist placed the $\mathrm{P}-\mathrm{N}$ $\mathrm{L}-\mathrm{N}$ or microcoil in or near the SPNs in the CT room $2 \mathrm{~h}$ before surgery. First, based on the location of the SPNs, the appropriate positioning of the patient was selected, and the puncture route was marked on the body surface. After local

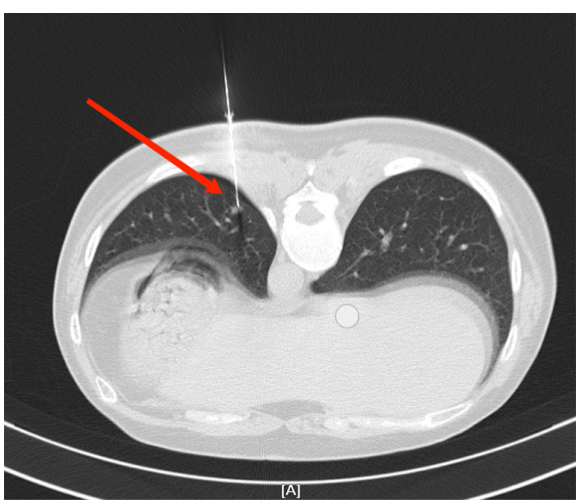

Figure 3 Localization of a nodule using a microcoil. The red arrow shows the location of SPNs. SPNs, small pulmonary nodules.

anesthesia with $2 \%$ lidocaine, the P-N-L-N was inserted along the designated puncture path, according to the standard procedure for P-N-L-N (10). Finally, CT scanning was performed to observe whether the anchor positioning was satisfactory (Figure 2). Using a similar approach, a suitable microcoil was used for percutaneous localization of SPNs. The needle tip was positioned in the normal lung parenchyma around the SPN, and the microcoil was inserted through the puncture needle (Figure 3).

\section{Surgery}

Patients who underwent preoperative localization proceeded to surgery on 2 hours later. VATS was performed under intravenous anesthesia and local block (Figure 4), using a laryngeal mask instead of endotracheal intubation and maintaining spontaneous ventilation (Figure 5). According to our operative procedures $(11,12)$, wedge resection or segmentectomy or lobectomy was performed based on the location and size of the SPNs (Figure 6). After the thoracic surgeon resected the affected lung tissue, the tumors in the lung tissue samples were located and labeled with silk sutures (Figure 7), before the specimens were sent to the pathology department. If the pathological diagnosis was malignant, radical resection of the lung cancer was performed (13).

\section{Observational indexes}

The operative time were from the beginning of the patient's skin cutting to the end of the skin suturing, the intraoperative blood loss were the volume of all fluid in 


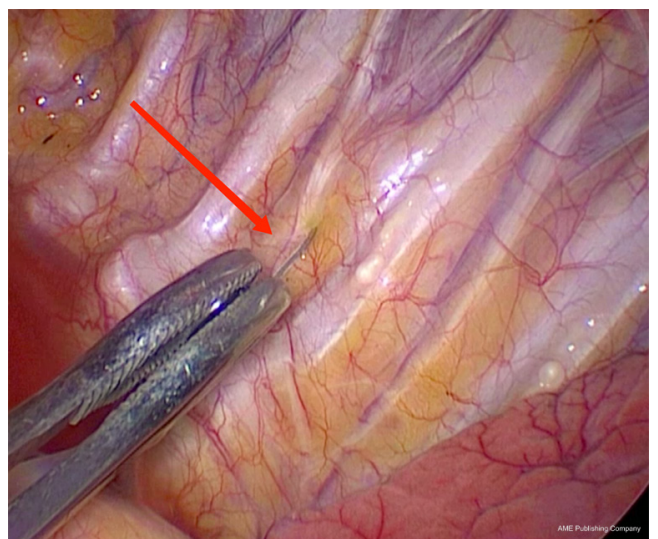

Figure 4 Intercostal nerve block using ropivacaine. The red arrow indicates the intercostal nerve.

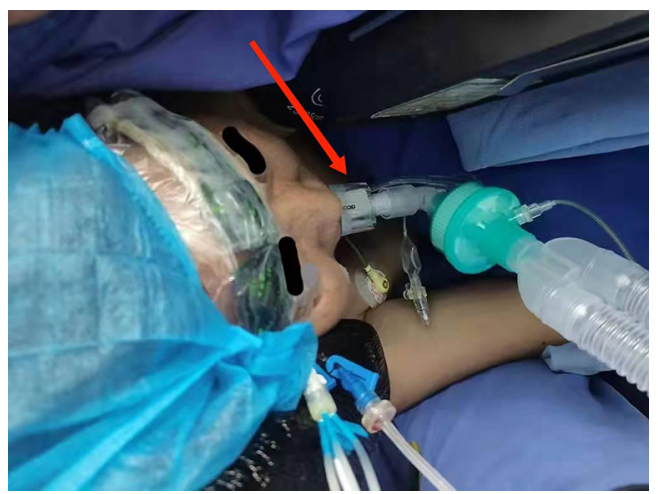

Figure 5 The laryngeal mask is used instead of an endotracheal tube for ventilation. The red arrow indicates the laryngeal mask. This image is published with the patient's consent.

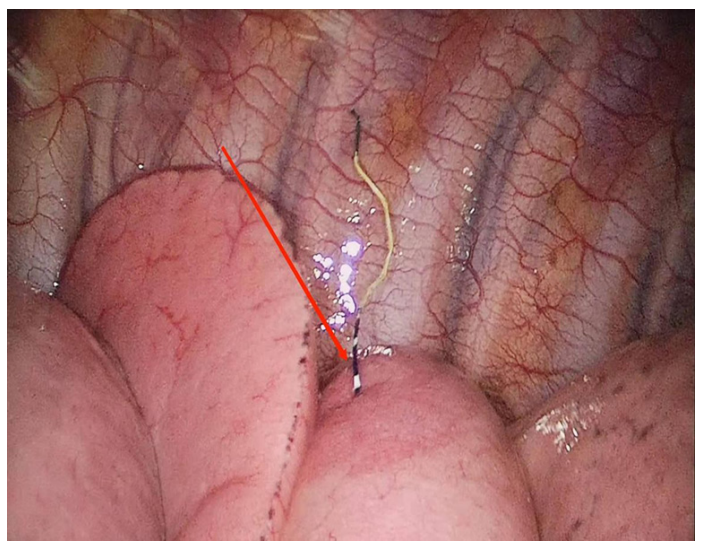

Figure 6 The P-N-L-N seen during operation. The red arrow indicates the tail of the P-N-L-N that is easy to see. P-N-L-N, pulmonary-nodule-location-needle.

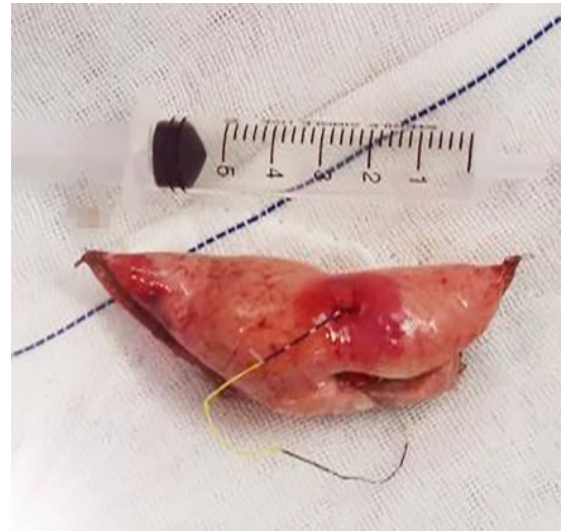

Figure 7 The specimen of the lung after wedge resection.

container connected to aspirator during surgery minus the fluid that irrigates chest, they were all from the anesthesia $\log$ sheet. The number of postoperative drainage days were from the first day after the patient's surgery to the chest tube was removed after the patient has no pneumothorax on chest X-ray and the chest drainage flow is less than $150 \mathrm{~mL}$ per day. The postoperative total drainage volume were the total amount of chest drainage from the first day after surgery until the chest tube was removed. The postoperative discharge time were from the first day after surgery to the discharge time of patients with normal chest X-ray, blood routine examination and biochemical indicators. The number of postoperative drainage days, postoperative total drainage volume, postoperative discharge time were from nursing record, the pathological diagnosis were from report of postoperative pathological examination diagnosed by pathology department. We also recorded the number of cases in the localization group in which localization materials were missing, displaced, or retained in lung tissue during surgery.

\section{Statistical analysis}

The data in this study were analyzed by SPSS 22.0 statistical software. Measurement data were expressed as mean $\pm \mathrm{SD}$, enumeration data were compared using $\chi^{2}$ test; analysis of variance was used for comparison between groups and the least significant difference-t test was used for pair comparison. In addition, multiple regression analysis is used for comparison between P-N-L-N and Microcoil groups. The test level was $\alpha=0.05$, and $\mathrm{P}<0.05$ was considered 
Table 2 Comparison of indicators between the localization and unlocalized groups

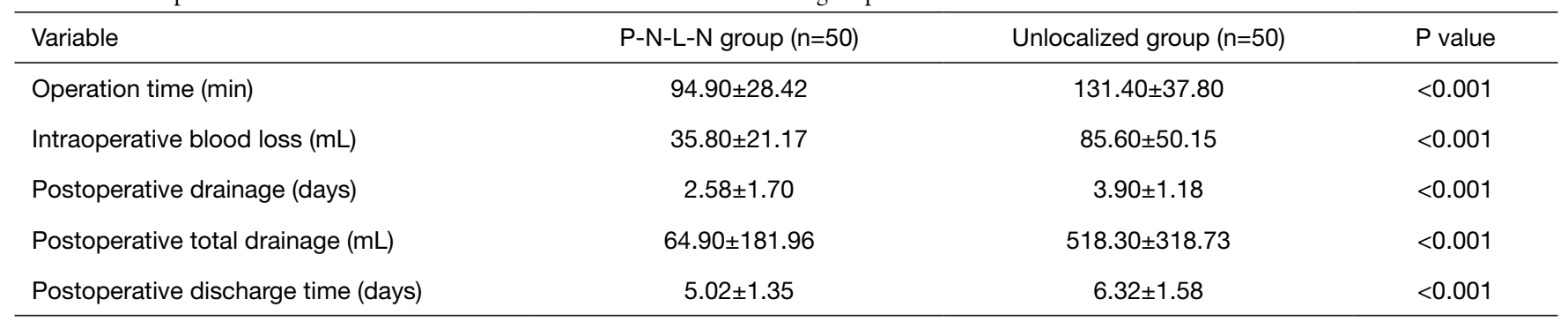

P-N-L-N, pulmonary-nodule-location-needle.

Table 3 Comparison of indicators between the localization and unlocalized groups

\begin{tabular}{|c|c|c|c|}
\hline Variable & Microcoil group $(n=50)$ & Unlocalized group $(n=50)$ & $P$ value \\
\hline Intraoperative blood loss (mL) & $75.00 \pm 65.22$ & $85.60 \pm 50.15$ & 0.35 \\
\hline Postoperative drainage (days) & $3.18 \pm 2.49$ & $3.90 \pm 1.18$ & 0.10 \\
\hline Postoperative total drainage $(\mathrm{mL})$ & $648.52 \pm 708.81$ & $518.30 \pm 318.73$ & 0.27 \\
\hline
\end{tabular}

statistically significant.

\section{Results}

Based on the inclusion and exclusion criteria, we retrospectively collected 100 cases of SPNs in the localized group (50 cases in the P-N-L-N group, 50 cases in the microcoil group) and 50 cases in the unlocalized group. In the P-N-L-N group, inability to identify the localization needle or its displacement and residual in the lung tissue was not excluded. However, in the microcoil group, 1 patient had a microcoil that could not be found in the lung tissue, 3 patients had a microcoil that could not be clearly identified during surgery, and 3 patients had microcoil displacement, these 7 patients were excluded.

CT-guided puncture of SPNs and placement of a $\mathrm{P}-\mathrm{N}-\mathrm{L}-\mathrm{N}$ before pulmonary resection during nonintubated VATS significantly reduced the operative time, intraoperative blood loss, drainage tube placement time, postoperative total drainage volume, and postoperative discharge time, as shown in Table 2.

CT-guided puncture of SPNs and placement of a microcoil before pulmonary resection during non-intubated VATS significantly reduced the operative time, as are shown in Table 3.

CT-guided puncture of SPNs before pulmonary resection and placement of either a P-N-L-N or a microcoil can reduce the operative time, intraoperative blood loss, and postoperative total drainage volume, as shown in Table 4.

\section{Discussion}

Enhanced recovery after surgery (ERAS) and the concept of holistic minimally invasive surgery have become hot topics in thoracic surgery. Both advocate optimizing the anesthesia method, reducing surgical trauma and controlling the stress response to reduce the injury caused by surgery and anesthesia (14). At present, the popularization of the VATS pulmonary resection technique has led to its widespread use in clinical practice and it is the first choice for the treatment of early lung cancer (15). Compared with thoracotomy, its advantages are less trauma, short operation time, quick recovery and fewer surgical complications (16). Doublelumen or single-lumen endotracheal intubation single-lung ventilation general anesthesia has been the main anesthesia method for VATS, but both endotracheal intubation and general anesthesia often have related complications (17) such as acute lung injury and arrhythmia, which may delay postoperative recovery, prolong postoperative hospitalization time and increase hospitalization cost (18). In recent years, based on the requirements of ERAS and holistic minimally invasive surgery, the non-tracheal intubation anesthesia 
Table 4 Comparison of indicators between the P-N-L-N and microcoil groups

\begin{tabular}{|c|c|c|c|}
\hline Variable & P-N-L-N group $(n=50)$ & Microcoil group $(n=50)$ & $P$ value \\
\hline Intraoperative blood loss (mL) & $35.80 \pm 21.17$ & $75.00 \pm 65.22$ & $<0.001$ \\
\hline Postoperative drainage (days) & $2.58 \pm 1.70$ & $3.18 \pm 2.49$ & 0.16 \\
\hline Postoperative total drainage $(\mathrm{mL})$ & $64.90 \pm 181.96$ & $648.52 \pm 708.81$ & $<0.001$ \\
\hline
\end{tabular}

P-N-L-N, pulmonary-nodule-location-needle.

technique has been advocated and popularized (19).

In recent years, non-tracheal intubation anesthesia has developed rapidly, and preservation of the patient's selfbreathing during thoracoscopic surgery can effectively avoid the occurrence of airway trauma and postoperative complications caused by tracheal intubation $(20,21)$. In our team's previous study (4), performing VATS pneumonectomy under non-intubation anesthesia reduced the intraoperative blood loss, postoperative drainage, postoperative white blood cell and neutrophil proportions, and reduced hospitalization costs. It has been reported that endotracheal intubation can cause sympathetic nerve excitation (22), which leads to increased blood pressure, whereas the non-endotracheal intubation method avoids the pressure effect, and hemodynamics remain more stable. In addition, surgical trauma or tracheal intubation can lead to an acute inflammatory response in the patient, producing C-reactive protein (CRP), interleukin-6 and other inflammatory factors (23). It has been reported that the CRP level significantly decreased after non-endotracheal intubation anesthesia pneumonectomy (24), which suggests that it can reduce surgical or tracheal intubation trauma, reduce the level of inflammatory factors and inflammatory response, and thus reduce the use of antibiotics, shorten the length of hospital stay and reduce the cost of hospital stay.

However, during thoracic surgery under non-tracheal intubation anesthesia, the patient's end-tidal carbon dioxide $\left(\mathrm{EtCO}_{2}\right)$ will increase, which will have adverse effects on the patient's physiological response and the longer the operation time, the greater the adverse effects on the patient $(25,26)$. Shorter operation time is better for rapid postoperative recovery (27). In order to reduce the operation time and for a smooth operation under non-endotracheal anesthesia, it is necessary to locate the SPNs quickly. Therefore, preoperative localization of SPNs is required.

There are various localization methods (28). The most commonly used traditional positioning method is palpation, whereby the surgeon intraoperatively determines the location of the SPNs by touching and pressing with a finger or instrument to identify the difference in density between the SPNs and the surrounding lung tissue. However, for some SPNs with a small diameter and at a long distance from the pleural edge, palpation may not accurately identify their location (29). In addition, due to deflation of the lung during surgery, it can be difficult to accurately detect and localize SPNs. It has been reported that the incidence of changing from VATS to thoracotomy is high when there is inaccurate localization or recognition of SPNs (30). In addition, in the resected specimens, palpation is not conducive to quickly and accurately finding the SPNs, which can also prolong the pathological examination time. In this study, the duration of surgery was shorter in the localization group than in the unlocalized group, confirming that preoperative localization of SPNs is essential to reduce the operation time.

Ultrasonic positioning has been proposed (31), but for patients with asthma, pulmonary bullae, diffuse emphysema and pulmonary fibrosis, the resolution of ultrasound is significantly reduced due to insufficient collapse of lung tissue and presence of gas in the lung. Other methods also proposed are injection of methylene blue, medical glue and other contrast agents into the SPNs under CT guidance (32). With these methods, the tissue around the nodules may appear faint, and the boundary between the nodules and surrounding tissues is not clear. The use of medical glue may cause irritant cough in some patients (33). Hook-wire (34), spring coil (35) and other positioning devices placed under CT guidance have been described, but complications such as pneumothorax, bleeding and dropping of positioning needles can occur.

In this study, P-N-L-N and microcoil were used for preoperative positioning of SPNs. For patients with 
microcoil positioning, the thoracic surgeon has to use instrument palpation to identify its exact position, so there is more traction on lung tissue, which may cause certain damage to the lung tissue, leading to some exudation and increased postoperative drainage and a longer postoperative hospital stay. Therefore, the total postoperative drainage volume, the number of days of drainage and the length of postoperative hospital stay were not statistically significant compared with the unlocalized group.

Compared with the microcoil, the attachment line of the P-N-L-N has different color that makes it easier to observe both during the operation and in the resected specimen. Therefore, using this positioning method to locate SPNs can reduce the operation time even more. This benefit is especially powerful for surgery performed under non-tracheal intubation anesthesia. Accurate preoperative localization of SPNs can ensure precise removal of the nodule and reduce the lung resection margin during surgery, which preserves as much lung tissue of the patient as possible, not only improving postoperative lung function, but also reducing intraoperative blood loss, and postoperative total drainage, as well as accelerating the postoperative recovery of patients. In addition, special hook structure in the front end of the P-N-L-N can be firmly anchored in the lung tissue, thus reducing complications such as displacement and decoupling during postural changes, respiratory movements, and surgical traction (36).

According to the latest expert consensus (37), patients can get out of bed early and eat as soon as possible after SPN resection with non-intubation anesthesia. Removal of SPNs is particularly suitable for non-intubated VATS because most are early-stage tumors requiring only sublobectomy. Preoperative positioning of SPNs is more conducive to non-intubated VATS and conforms to the concept of rapid recovery. Moreover, pneumonectomy with non-intubated VATS has the same oncologic significance as tracheal intubation anesthesia (38).

\section{Study limitations}

Non-endotracheal intubation anesthesia also has challenges such as the effects of elevated $\mathrm{EtCO}_{2}$ on patients, hypoxemia and large mediastinal oscillations (39). In addition, CT-guided localization with the $\mathrm{P}-\mathrm{N}-\mathrm{L}-\mathrm{N}$ is an invasive procedure, leading to some complications such as pleural reaction, pneumothorax and intrapulmonary hemorrhage (40). There are also some patients with SPNs that cannot be located percutaneously, because localization is prevented by the patient's ribs.

\section{Future directions}

Further research is needed on the physiological effects of elevated $\mathrm{ETCO}_{2}$ on patients undergoing non-intubated VATS, and improvements are needed to overcome the effects of greater respiratory mobility on surgical operations. For the positioning method, some researchers have proposed the use of electromagnetic guided bronchoscopy (41), which is less traumatic and can reduce the displacement and shedding of markers caused by respiratory movement (42). However, at present, that method is expensive and the positioning time is long. Therefore, we also need to further explore better non-invasive ways of preoperative positioning for non-intubated VATS.

\section{Conclusions}

In summary, preoperative localization of SPNs is conducive for VATS under non-intubated anesthesia, and the effect of positioning by $\mathrm{P}-\mathrm{N}-\mathrm{L}-\mathrm{N}$ is better than with the microcoil.

\section{Acknowledgments}

Funding: The study was supported by Science and Technology Program of Kunming City (2020-1-H-003), the opening project of Chest Disease Clinical Medical Center of Yunnan First People's Hospital in 2021 (2021LCZXXFXB03), Yunnan Clinical Medical Center for Respiratory Diseases (2021LCZXXF-HX02).

\section{Footnote}

Reporting Checklist: The authors have completed the STROBE reporting checklist. Available at https://jtd. amegroups.com/article/view/10.21037/jtd-22-114/rc

Data Sharing Statement: Available at https://jtd.amegroups. com/article/view/10.21037/jtd-22-114/dss

Conflicts of Interest: All authors have completed the ICMJE uniform disclosure form (available at https://jtd.amegroups. com/article/view/10.21037/jtd-22-114/coif). The authors have no conflicts of interest to declare.

Ethical Statement: The authors are accountable for all aspects of the work in ensuring that questions related 
to the accuracy or integrity of any part of the work are appropriately investigated and resolved. Ethical approval for the study was granted by the Ethics Committee of The First People's Hospital of Yunnan Province (No. KHLL2021-KY091). In addition, written informed consent was obtained from all patients who participated in the study. All procedures performed in this study involving human participants were in accordance with the Declaration of Helsinki (as revised in 2013).

Open Access Statement: This is an Open Access article distributed in accordance with the Creative Commons Attribution-NonCommercial-NoDerivs 4.0 International License (CC BY-NC-ND 4.0), which permits the noncommercial replication and distribution of the article with the strict proviso that no changes or edits are made and the original work is properly cited (including links to both the formal publication through the relevant DOI and the license). See: https://creativecommons.org/licenses/by-nc-nd/4.0/.

\section{References}

1. Kong J, Guo J, Zhang H, et al. CT-guided localization techniques of small pulmonary nodules: a prospective non-randomized controlled study on pulmonary nodule localization needle and methylene blue staining with surgical glue. J Thorac Dis 2020;12:6826-35.

2. Zhou J, Ai M, Xu J. CT-guided systematic management of solitary pulmonary nodules. Military Medical Journal of Southeast China 2020;22:94-96.

3. Su L, Wei XQ. Diagnosis and treatment strategy of early lung cancer from the development of prevention and control of lung cancer in China. Journal of Medical Research 2020;49:1-5.

4. McLellan R, Marshall H, Dent A, et al. Diagnosis and treatment of early lung cancer. Aust J Gen Pract 2020;49:508-12.

5. Ali JM, Volpi S, Kaul P, et al. Does the 'non-intubated' anaesthetic technique offer any advantage for patients undergoing pulmonary lobectomy? Interact Cardiovasc Thorac Surg 2019;28:555-8.

6. Lan L, Jiang L, Duan C, et al. A risk score for predicting postoperative complications in non-intubated thoracic surgery. J Thorac Dis 2021;13:3960-8.

7. Nelson DB, Antonoff MB. Commentary: Revisiting old data: Is video-assisted thoracoscopic surgery still superior to thoracotomy when enhanced recovery is applied? J Thorac Cardiovasc Surg, 2019. [Epub ahead of print]. doi: 10.1016/j.jtcvs.2019.08.095.

8. Nardini M, Dunning J. Pulmonary nodules precision localization techniques. Future Oncol 2020;16:15-9.

9. Liu J, Wang X, Wang Y, et al. Comparison of CT-guided localization using hook wire or coil before thoracoscopic surgery for ground glass nodules. Br J Radiol 2020;93:20190956.

10. Chen ZM, Xu JY, Cai WQ, et al. The 4-hook anchor coaxial needle with scaled suture is superior to the double spring coil for preoperative localization. J Thorac Dis 2021;13:4455-63.

11. Peng J, Chen XL, Mao X, et al. Video-assisted thoracoscopic right lower lobectomy for lung cancer using the Harmonic scalpel. J Thorac Dis 2013;5:864-7.

12. Peng J, An S, Wang HP, et al. Video-assisted thoracoscopic surgery lobectomy for lung cancer versus thoracotomy: a less decrease in sVEGFR2 level after surgery. J Thorac Dis 2016;8:323-8.

13. Peng J, Chen XL, Mao X, et al. Video-assisted thoracoscopic left upper lobe apical trisegmentectomy with the Harmonic scalpel. J Thorac Dis 2014;6:1822-5.

14. Ni HX, Zhao W, Hu JH, et al. The clinical application of CT-guided microcoil positioning of small pulmonary nodules in video-assisted thoracic surgery. Journal of Interventional Radiology 2017;26:555-559.

15. Ferlay J, Soerjomataram I, Dikshit R, et al. Cancer incidence and mortality worldwide: sources, methods and major patterns in GLOBOCAN 2012. Int J Cancer 2015;136:E359-86.

16. Zhao H, Bu L, Yang F, et al. Video-assisted thoracoscopic surgery lobectomy for lung cancer: the learning curve. World J Surg 2010;34:2368-72.

17. Yang ZX, Zhang DG, Dong YJ, et al. Single hole thoracoscopy combined with Hook-wire needle positioning technology under non tracheal intubation anesthesia Clinical application of GGO in peripheral lung lesions. Journal of Henan University(Medical Science) 2020;39:419-23.

18. Son SL, Kim WS, Kwon M, et al. Airway obstruction during pneumonectomy using a single lumen tube: A case report. Medicine (Baltimore) 2020;99:e19736.

19. Poon B, Seliman H. ERAS \& Tubeless-Michael Haddock: the past and future of proton beam. Chin Clin Oncol 2017;6:56.

20. Bao L, QU MY, Xiao ZY. Research progress of laryngopharyngeal complications after tracheal intubation general anesthesia. Journal of Clinical Anesthesiology 2018;34:501-4. 
21. Yoshimura K, Ichioka K, Terada N, et al.

Retroperitoneoscopic tubeless cutaneous ureterostomy. BJU Int 2002;89:964-6.

22. Huang HQ, Liu HZ, Wang HB, et al. Application of paravertebral nerve block combined with laryngeal mask general anesthesia in excision of unilateral bullae. Chongqing Medicine 2019;48:1716-9.

23. Wu JX, Xu MY. Fast-track anesthesia in thoracic surgery. Shanghai Medical Journal 2020;43:67-71.

24. Wen J, Duan N, Li XG, et al. Efficacy of intubated and non-intubated anesthesia in uniportal thoracoscopic surgery for thoracosympathectomy. Journal of Shanxi Medical University 2017;48:296-300.

25. Liu Z, Yang R, Sun Y. Tubeless uniportal thoracoscopic wedge resection with modified air leak test and chest tube drainage. BMC Surg 2020;20:301.

26. Shi Q, Diao Y, Qian J. Application of Single-hole Thoracoscopic Surgery Combined with ERAS Concept for Respiratory Function Exercise in Perioperative Period of Lung Cancer. Zhongguo Fei Ai Za Zhi 2020;23:667-72.

27. Kim NH, Kang SJ. Correlation between the Time to Surgery and That to Recovery from Postoperative Diplopia Based on a Single-Center, Retrospective Experience: A Case Series of 11 Patients. Arch Plast Surg 2014;41:486-92.

28. Yoshida R, Yoshizako T, Tanaka S, et al. CT-guided color marking of impalpable pulmonary nodules prior to video-assisted thoracoscopic surgery. Clin Imaging 2021;74:84-8.

29. Li SP, Wang L. Current status of research on location of pulmonary nodules. Journal of Clinical Medical Literature (ElectronicEdition) 2020;7:191-2.

30. Hu CG, Zheng K, Liu GH, et al. Effectiveness and postoperative pain level of single-port versus twoport thoracoscopic lobectomy for lung cancer: a retrospective cohort study. Gen Thorac Cardiovasc Surg 2021;69:318-25.

31. Manca G, Davini F, Tardelli E, et al. Clinical Impact of Radioguided Localization in the Treatment of Solitary Pulmonary Nodule: A 20-Year Retrospective Analysis. Clin Nucl Med 2018;43:317-22.

32. Wang L, Wu WH, Hu DZ, et al. A primary study of intraoperative ultrasound location of pulmonary groundglass opacities in video-assisted thoracic surgery. Chinese Journal of Ultrasonography 2018;27:293-6.

33. Chao YK, Pan KT, Wen CT, et al. Preoperative CT versus intraoperative hybrid DynaCT imaging for localization of small pulmonary nodules: a randomized controlled trial. Trials 2019;20:400.

34. Fan QH, Wu XM, Wang JF, et al. A clinical comparative study of CT-guided hardening agent localization before the thoracoscopic surgery of small pulmonary nodules. China Modern Doctor 2017;55:16-9.

35. Adachi H, Sakamaki K, Nishii T, et al. Lobe-Specific Lymph Node Dissection as a Standard Procedure in Surgery for Non-Small Cell Lung Cancer: A Propensity Score Matching Study. J Thorac Oncol 2017;12:85-93.

36. Huo SQ, Cai WQ, Liao FC, et al. CT-guided localization of small pulmonary nodule with new pulmonary nodule locating needle before thoracoscopic surgery. Chinese Journal of Interventional Imaging and Therapy 2020;17:667-70.

37. He J, Liang H, Wang W, et al. Tubeless video-assisted thoracic surgery for pulmonary ground-glass nodules: expert consensus and protocol (Guangzhou). Transl Lung Cancer Res 2021;10:3503-19.

38. Prisciandaro E, Bertolaccini L, Sedda G, et al. Nonintubated thoracoscopic lobectomies for lung cancer: an exploratory systematic review and meta-analysis. Interact Cardiovasc Thorac Surg 2020;31:499-506.

39. Liu LL, Xu N, Hu YY, et al. Research progress of videoassisted thoracoscopic surgery with preservation of spontaneous breathing and non-endotracheal intubation anesthesia. Journal of Clinical Pulmonary Medicine 2020;25:145-8.

40. Xu MX, Zhao ZK, Liang Y, et al. Application and complication analysis of CT guided wire with hook for precise localization of pulmonary ground glass nodules before thoracoscopy. Journal of China Clinic Medical Imaging 2020;31:65-7.

41. Zhang JS, Chen EG. Application of the whole lung virtual navigation system in clinical practice. Modern Practical Medicine 2020;32:4-6.

42. Cui X, Mu F, Qin Y, et al. Development of Magnetic Anchoring Lung Nodule Positioning Device. Zhongguo Yi Liao Qi Xie Za Zhi 2021;45:32-6.

(English Language Editor: K. Brown)

Cite this article as: Yu Y, Liu RS, Bo Y, Ye J, Huang Y, Xu ZZ, Yang ZY, Chen ZM, Peng J. Comparison of two preoperative positioning techniques and unpositioning methods in nonintubated video-assisted thoracoscopic surgery. J Thorac Dis 2022;14(2):464-473. doi: 10.21037/jtd-22-114 